\title{
NUEVOS ENFOQUES TEÓRICOS PARA EL ESTUDIO DE LA POESÍA LÍRICA
}

\author{
NEW THEORETICAL APPROACHES \\ TO THE STUDY OF LYRIC POETRY
}

Isabel GONZÁLEZ GIL

Universidad Complutense de Madrid

migonzalezgil@ucm.es

\begin{abstract}
Resumen: Este artículo examina dos modelos teóricos recientes y contrapuestos de estudio del género lírico: el mimético-narratológico y el performático, que constituyen sendas aportaciones de la crítica literaria internacional al respecto en las dos últimas décadas, pero que han tenido escasa difusión en la teoría literaria en lengua española. Se trata, por un lado, de las aplicaciones de la narratología al estudio de textos poéticos y líricos y, por otro lado, la propuesta de Jonathan Culler en su Theory of the Lyric, que rechaza la asimilación de la lírica al género narrativo y propone indagar en su especificidad, no mimética sino performática.
\end{abstract}

Palabras clave: Teoría de la lírica. Narratología transgenérica. Poesía narrativa. Performance.

Abstract: This paper examines two recent and opposite models for the study of the lyric genre: mimetic-narratological and performative, two important contributions of international literary criticism to the field. Nevertheless, they have had scarcely any repercussions in Spanish literary theory. First, we focus on the narratological applications to the study of poetic and lyric texts. Second, we address Jonathan Culler's theoretical proposal developed in his book Theory of the Lyric, in which he rejects the assimilation of lyric poetry to the model of narrative fiction, and he proposes instead to investigate its singularity, not imitative but performative. 
Key Words: Theory of the lyric. Transgeneric narratology. Narrative poetry. Performance.

\section{INTRODUCCIÓN}

La poesía lírica ha ocupado una extraña posición en las universidades en las últimas décadas, a un tiempo privilegiada y marginal: como diagnosticaba Garrido Gallardo en su entrevista con Sofía M. Carrizo, "hoy la Lírica es considerada la archiliteratura: en cierto sentido, la matriz teórica de la literariedad" (Carrizo, 2014: 139); pese a ello, lamentaba Ángel Luján, la lectura de poesía ha ido progresivamente reduciéndose a una actividad de especialistas y poetas; temida a la par que venerada por el público general de estudiantes, al transformarse "en un terreno casi sagrado al que un respeto exagerado impide el acercamiento" (2005: 12).

Mientras la narrativa y el teatro desarrollaron marcos teóricos ad hoc para el género en la segunda mitad del pasado siglo, como fueron la narratología y la semiótica teatral, la lírica no alcanzó un marco de estudio unitario y actualizado, a pesar de que tanto el formalismo como el estructuralismo prestasen al género una especial atención en sus investigaciones sobre el lenguaje literario y de las importantes aportaciones de la lingüística, especialmente de la semántica estructural, al análisis textual; sino que, como señalaba Pozuelo Yvancos, su investigación "ofrece en la Poética contemporánea una enorme dispersión, cuando no cierto anquilosamiento" (1994: 177) ${ }^{1}$. Los estudios de poesía siguen adoleciendo en cierta medida de lo que reprochaba García Berrio hace cuatro décadas: "nuestro conocimiento del poema analizado, como realidad global literaria, no mejora casi en nada al que nos proporcionaban los útiles de los métodos clásicos" (1978: 22)2. El investigador o doctorando que decide estudiar

\footnotetext{
${ }^{1}$ Este anquilosamiento "contrasta en el conjunto de la teoría con el vigor alcanzado por la Narratología y con la creciente afirmación de la Semiología dramática. No me refiero, cuando diagnostico esta situación, a órdenes cuantitativos; al contrario, son muchos los libros dedicados al análisis de la poesía, pero adolecen casi todos, con notables excepciones, de las limitaciones de orden cualitativo: en primer lugar, su enorme dispersión en conjuntos de estudios sobre poetas y poemas, sin que se traduzca casi nunca una consideración de Poética general" (Pozuelo, 1994: 177). 2 "Piénsese si no ha sido espectacular el desarrollo de la que a veces se llama 'narratología'. Obviamente, por el olvido parcial de los cultivadores de la Poética en los últimos años y, sobre todo, dado el largo camino recorrido por la tradición retórica, los resultados en el análisis de los poemas líricos no han podido ser tan espectaculares. Los descubrimientos de quienes — pocos en
} 
una obra poética se encuentra confrontado con diversos marcos teóricocríticos, no siempre conciliables en cuanto a terminología y metodología de análisis. Esta carencia repercute también en los niveles preuniversitarios, en los que el comentario de texto lírico pervive como un ejercicio deudor de los repertorios tradicionales de figuras retóricas, acompañado de algunas nociones de crítica (como pueden ser las de sujeto lírico, tema, estructura, etc.) y de su relación con el contexto. Las aportaciones que desde la Teoría de la literatura han realizado en España figuras como la de García Berrio (1989), Pozuelo Yvancos (1998, 2009) o Luján (2005), han contribuido a colmar esa carencia, sin que ninguna se haya consolidado como un marco general para el estudio del género. A ellas se han sumado compilaciones como las de Fernando Cabo: Teoría del poema: la enunciación lírica, y Teorías sobre la lírica, en la que traduce al castellano algunos de los principales textos sobre la materia, que dan cuenta del panorama tan heterogéneo que existe, así como de los principales focos de desacuerdo.

En otros contextos académicos, la situación teórica de la poesía ha sido objeto también de preocupación. Como observaba el teórico alemán Peter Hühn:

The theoretical foundations of poetry criticism are increasingly being deplored as deficient. [...] The analytical categories and procedures of poetry interpretation have been criticised as largely intuitive, eclectic and lacking systematic organisation (2005: 147).

En las universidades estadounidenses el género lírico no ha encontrado tampoco un más amplio consenso en sus aspectos teóricos. Como advierte Jonathan Culler en su artículo "Why lyric?", "narrative has become the norm of literature" (2014: 201). El género narrativo se ha convertido en el dominante en unos estudios literarios centrados principalmente en la prosa de ficción ${ }^{3}$. Este es uno de los motivos por

verdad - han pretendido, con ignorancia u olvido de lo muchísimo conseguido por la tradición en el análisis de la lírica, difundir alguna técnica pretendidamente revolucionaria, no han engañado la sensatez de la mayoría de los profesionales de esta ciencia. Tras alardes terminológicos y de formalización, en cualquier caso menos precisos que los conocidos y olvidados desde la tradición retórica del comentario de textos, la potencia explicativa de tales modelos no supera la del análisis tradicional" (García Berrio, 1978: 22).

${ }^{3}$ En su Theory of the Lyric, Culler achaca a la falta de una teoría adecuada de la lírica el desplazamiento que sufre actualmente en los estudios literarios: "Lyric was once central to the experience of 
los que el estudio del texto lírico, según su parecer, ha tendido a ser progresivamente asimilado al modelo mimético de la ficción narrativa. En esta asimilación radica para Culler el principal error de enfoque de las teorías actuales de la lírica. Así, su propuesta de un nuevo marco no mimético de estudio busca configurar unas nuevas coordenadas que permitan aprehender aquello que diferencia al género.

Al mismo tiempo, la poesía ha constituido un punto ciego (Brian McHale) en los estudios de narratología, a pesar de que la mayor parte de las obras narrativas en la literatura occidental son formalmente poemas, y de la propia tradición de la Poética, vinculada a los poemas homéricos desde sus primeras reflexiones. Este descuido puede relacionarse con diversas causas, entre las que McHale destaca la especialización de los programas y los investigadores. Por ello, la especificidad de la poesía narrativa permanece, si cabe, aún más desatendida.

A la falta de una fundamentación teórica se le han sumado las dudas sobre la conveniencia del término lírica y de la existencia misma del género, que entre los críticos contemporáneos sigue siendo un objeto de controversia. Ya décadas atrás, René Wellek, en 1967, o T. S. Eliot, en 1953, recomendaban abandonar los intentos de definir el género por no encontrar ningún rasgo que pudiera convenir plenamente a sus diversas manifestaciones históricas y culturales ${ }^{4}$.

La corriente denominada New Lyric Studies, encabezada por las teóricas norteamericanas Virginia Jackson $(2005,2008)$ y Yopie Prins (2014), ha defendido que la lírica es, más que un género, una manera de leer que empezaría a gestarse en el Romanticismo, periodo en el que comenzaría el colapso de los distintos subgéneros líricos, hacia una idea

\footnotetext{
literature and to literary education, but it has been eclipsed by the novel, perhaps in part because we lack an adequate theory of the lyric. Even in the age of high theory, despite de interest in the linguistic analysis of poetic language, theoretical accounts of the lyric tended to be negative ones, designed to contrast with the real objects of theoretical interest. From Mikhail Bakhtin, who treated lyric as monological, in contrast with the rich dialogism of the novel, to Roland Barthes, for whom lyric sought to destroy language rather than engage with it [...], important theorist failed to develop a rich theoretical discourse about lyric, or of poetry in general, for that matter" (2015:3).

${ }^{4}$ Eliot: "the word cannot be satisfactorily defined"; Wellek: "La vía de salida es evidente. Se debe abandonar todo intento de definir la naturaleza general de la lírica o lo lírico. De ello no pueden resultar nada más que generalidades del tipo más trillado. Parece mucho más rentable enfocar el estudio hacia la variedad de la poesía y su historia y de este modo hacia la descripción de géneros que pueden aprehenderse en sus convenciones y tradiciones concretas" (Cabo, 1999: 53).
} 
más general y abstracta de la poesía ("lyricization”), y, posteriormente, debería su construcción crítica a los New Critics. No existiría, por tanto, un género lírico como tal, sino una lectura lírica de obras que anteriormente habían sido leídas según los códigos de los géneros a los que pertenecían (oda, elegía, etc.). Esta corriente teórica considera que el principal objeto de estudio de la Poética debería desplazarse del género hacia los subgéneros históricos y centrarse en investigar la genealogía de la conformación de dicha lectura lírica, a partir de la segunda mitad del siglo XVIII ${ }^{5}$.

Esta postura ha sido contestada por figuras como Stephen Burt (2016) o Jonathan Culler (2014, 2015), que abogan por la existencia de rasgos comunes del género, a pesar de su multiplicidad de formas: un sustrato invariante que permitiría vincular las formas modernas de la lírica con poetas de otros tiempos y culturas.

Entre las teorías contemporáneas de la lírica que defienden la posibilidad de un estudio del género se han abierto dos vías fecundas de investigación, cuyas principales aportaciones se tratarán en los apartados siguientes: en primer lugar, los estudios de poesía y lírica desde un modelo narratológico y, a continuación, un enfoque como el de Jonathan Culler, que rechaza su equiparación con la narrativa y propone repensar la lírica desde la performance.

\section{NARRATOLOGÍA APLICADA AL TEXTO LÍRICO}

Los primeros estudios que abordaron la poesía desde la narrativa datan, según Peter Hühn (2012), de los años ochenta. En el ámbito alemán, los trabajos de Klaus D. Seemann (1984) y Menno Kraan (1991) investigaban las distintas formas de mediación que pueden aparecer en el poema: Seemann distinguía entre cinco niveles diferentes de comunicación, desde el autor biográfico hasta los caracteres, considerando este último un nivel a menudo inexistente en poesía, y el primero irrelevante (Hühn,

\footnotetext{
${ }^{5}$ Se pregunta Stephen Burt en su reseña del libro de Jackson y Prins, The Lyric Theory Reader: A Critical Anthology: "What is the New Lyric Studies, and why does it seem - to some of us - so disturbing and so hard to avoid, so misleading, so important, and so useful? The term refers to ideas set forth, or at least implied, by Yopie Prins in Victorian Sappho (1999); codified by Virginia Jackson in Dickinson's Misery (2005); summarized in a 2008 special issue of PMLA, in Jackson's long entry for "lyric" in the new Princeton Encyclopedia of Poetry and Poetics (2012), and in the forewords and head notes to the present volume" (2016: 422).
} 
2013: en línea). Kraan diferenciaba entre autor empírico, autor implícito y sujeto lírico, tres instancias cuya relación varía históricamente según los movimientos literarios ${ }^{6}$. Más adelante, Wolfgang Bernhart (1993) tratará sobre la perceptibilidad de la mediación en el poema, defendiendo la condición mediada en el poema pese a la ilusión de inmediatez producida (Hühn, 2013: en línea). También en la década de los 80, comienza a aplicarse la noción de trama a la poesía: Jack Stillinger en 1985 estudiaba "The Plots of Romantic Poetry", donde lamentaba la falta de aplicación de las nuevas categorías críticas a la poesía y reivindicaba que "poems have plots, characters, points of view, settings, and the rest just as regularly as works of prose fiction do" (1988: 209). Investigadores como Willem G. Weststeijn (1989), Jan van der Eng (1989) o Dore Levy (1988) también publicaron contribuciones de crítica poética desde este ángulo.

A pesar de estos estudios precursores en las últimas décadas del siglo XX, ha sido en las dos primeras décadas del siglo XXI cuando se han producido los principales avances y se han publicado las aportaciones mayores al respecto. Eva Müller-Zettelmann en 2002 planteaba la utilización de la narratología en los estudios sobre poesía. Müller-Zettelmann rechaza la caracterización tradicional de la lírica como el género de la subjetividad, que considera efecto de una ilusión estética producida por diversas técnicas (Hühn, 2013: en línea).

Los trabajos de Peter Hühn $(2004,2005,2012)$ han sido decisivos en el desarrollo de este enfoque. A principios de siglo comienza el SubProject P6, dirigido por Hühn y Jörg Schöner, dentro del Interdisciplinary Centre for Narratology (ICN) de la Universidad de Hamburgo ("The Theory and Methodology of the Narratological Analysis of Poetry: Approaches from the Perspective of English and German Studies"), cuyos resultados fueron publicados en el volumen editado en 2005 por Hühn y Jens Kiefer (The Narratological Analysis of Lyric Poetry: Studies in English Poetry from the 16th to the 20th).

Como afirman Hühn y Schönert (2005) en el apartado introductorio, el fundamento para el estudio narratológico de la poesía - y no solo de la poesía épica o con un componente narrativo, sino también la considerada

\footnotetext{
${ }^{6} \mathrm{Si}$ tradicionalmente ha sido un foco principal de discrepancia la existencia o no de mímesis y/o de ficción en el poema, el uso del término mediación, proveniente de la narratología y acuñado por Franz K. Stanzel (Alber y Fludernik, 2013), soslaya la problemática inscribiendo la lírica en un terreno compartido con la narrativa y el drama, que permite aplicar categorías transgenéricas.
} 
como puramente lírica- es que el texto lírico comparte con el narrativo sus tres aspectos fundamentales, que son la secuencialidad (sequentiality), la mediación (mediacy), que consiste en la selección, presentación e interpretación de una secuencia desde una determinada perspectiva, y la articulación expresiva (articulation); ya que en ambos géneros existe una secuencia temporal de eventos (mentales o psicológicos, habitualmente, en el caso de los textos líricos), se adopta una perspectiva, y se requiere de un acto de expresión. Es central para este enfoque la concepción de la narración como una práctica semiótica transgenérica:

The legitimacy of this approach depends on the premise that narration is an anthropologically universal semiotic practice, independent of culture and period, used to structure experience and produce and communicate meaning, and is as such one of the basic operations at work even in lyric poetry. If this is so, it is reasonable to assume that the well-developed precision and explanatory potential of modern narrative analysis — narratology - can help us conceptually refine and enhance the study of lyric poetry (Hühn y Schönert, 2005: 2).

Ese mismo año, Hühn publicaba el artículo "Plotting the lyric: forms of narration in poetry" en el volumen recopilatorio de Müller-Zettelman y Rubik (Theory into poetry: new approaches to the lyric), en el que aplica las categorías narratológicas al célebre poema de Wordsworth "I wandered lonely as a cloud". Dentro de la secuencialidad, Hühn emplea la noción de trama poética (poetic plot): "Plots in poetry are typically constituted by mental or psychological incidents such as perceptions, imaginations, desires, anxieties, recollections or emotions and their emergence and development" (Hühn 2005: 149).

Para el estudio de las tramas poéticas, adapta dos conceptos de la psicología cognitiva y de la lingüística: la noción de frame (en tanto que contextos temáticos o situacionales) y scripts (patrones secuenciales). La activación de dichos marcos (como referencia paradigmática al mundo externo y a esquemas previos, experienciales o literarios) es lo que permite al lector la interpretación del texto. A diferencia de la narrativa, señala Hühn, los poemas son habitualmente menos explícitos y circunstanciales, por lo que requieren una mayor implicación del lector para inferir dichos esquemas relevantes. 
En su introducción a The Narratological Analysis of Lyric, Schönert y Hühn complementaban estas dos nociones con las de desviación del esquema (schema-deviation), en cuanto que transgresión de las expectativas lectoriales (violation of expectations) $)^{7}$ y la aplicación al poema del concepto de suceso (event): "the decisive turning point in the sequence structure of a poem" (2005: 7). Dicho suceso puede tener lugar en el nivel de los acontecimientos, si está relacionado con uno de los caracteres en el plano de la historia; si, por el contrario, el cambio afecta al hablante o narrador, se trataría de un "presentation event", de un hecho discursivo.

En cuanto al segundo de los términos propuestos para el análisis narratológico de la lírica, el de mediación (mediacy), Hühn (2005) reduce a cuatro agentes la distinción por niveles de Seeman: el autor biográfico, el sujeto textual (variante del autor implícito), el hablante o narrador y el protagonista. Para Hühn, las referencias al autor empírico son relevantes como un criterio que permite determinar la plausibilidad histórica y cultural de los esquemas posibles (frames y scripts). Por otro lado, establece dos tipos diferentes de perspectiva en el poema: voz y focalización, aplicando las distinciones de Genette y narratólogos posteriores como Bal, Chatman o Fludernik, entre otros.

La traslación de estas categorías narratológicas al texto lírico permite a Hühn elucidar algunas especificidades del texto lírico, como serían tanto el colapso de dichos niveles de mediación, que en la poesía romántica crea una "illusion of immediacy, spontaneity and thus authenticity" (2005: 153) ${ }^{8}$, como su contrario, la enfática separación entre

\footnotetext{
${ }^{7}$ Esta noción de violación de las expectativas es especialmente importante para el estudio de la poesía lírica, sobre todo en la modernidad, ya que la transgresión ha sido considerada connatural al género por una parte importante de la crítica; incluso teóricos como Karlheinz Stierle (Cabo, 1999: 216) consideran la lírica como un anti-discurso, un discurso de la transgresión, donde se manifiesta en su esplendor la negatividad de Adorno; hasta el punto que una constante en el acercamiento teórico al género ha sido la de tratar de definirle por lo que no es. Stierle señala que "sobre la posibilidad de ser de la lírica incide desde el principio la circunstancia de que no se trata de un género propio, sino de un modo específico de transgredir un esquema genérico" (Cabo, 1999: 216). ${ }^{8}$ Esta corriente de la narratología aplicada al texto lírico insistirá en esta noción de ilusión de inmediatez frente a las teorías que abogan por la inmediatez de la lírica frente a la mediación narrativa. Según estas posturas, "el enunciado lírico tiene además una recepción distinta del enunciado de realidad. No hay ficción tampoco, puesto que la palabra tiene en el género lírico una función de 'inmediatez', frente al género ficcional cuya función es de 'mediación' y, en ese sentido, está al servicio de la mímesis" (Calles, 2002: 133-134). En cambio, para estos autores, dicha
} 
niveles, característica también de la poesía.

Según Hühn, las tramas líricas pueden ser categorizadas en función de su tipo ("thematic frame of development"), estructura ("dynamic impulse and plot mechanism"), relación protagonista-narrador (homodiegética o heterodiegética), acto de narración, función e intensidad (eventfulness); también según el tipo de evento y su lugar en el plano de la historia o del discurso (2005: 154), que permite discernir el momento central y los puntos de inflexión en el desarrollo de los mismos. Según la relación temporal entre narración e historia, Hühn propone tres tipos de trama: narración retrospectiva, simultánea y prospectiva, que pueden presentarse imbricadas.

Como señala el autor en su entrada para The living handbook of narratology ("Narration in Poetry and Drama"), lo que este enfoque narratológico de la poesía puede proporcionar es un método específico para analizar la estructura secuencial, así como un instrumento más preciso para distinguir en los poemas líricos los niveles y las formas de mediación (Hühn, 2012: en línea). También permitiría dar cuenta de la subversión de dicha secuencialidad, que ha sido uno de los rasgos más relevantes del género, o al menos de algunas de sus manifestaciones históricas, especialmente en la poesía moderna.

Otro de los focos importantes en el estudio narratológico de la poesía ha sido el proyecto de la Universidad North-West en Sudáfrica "Verse and narrative: narrative structures and techniques in lyric poetry", dirigido por Heilna du Plooy, Bernard Odendaal y Odil Heynders. En julio de 2009, organizaron un grupo de trabajo centrado en el estudio del uso de las estructuras y técnicas narrativas en textos líricos. De esa investigación nacieron dos volúmenes colectivos, uno sobre el corpus de la poesía escrita en afrikáans, y otro en inglés y neerlandés. Entre las conclusiones de du Plooy, se perfilaban diversos objetivos y líneas de investigación, como: el estudio del uso de las estructuras narrativas, la determinación de la prevalencia de los aspectos narrativos en la lírica contemporánea, la relectura de poesía de otros periodos históricos desde el estudio de la narratividad, la pregunta por las manifestaciones de la narratividad en la

inmediatez sería una ilusión, es decir, un efecto conseguido por un determinado procedimiento literario, un sistema de convenciones entre otros posibles. 
poesía lírica, la investigación acerca de la predominancia de lo narrativo en la poesía contemporánea y en la literatura posmoderna (el llamado giro narrativo), así como la adaptación de conceptos narratológicos para el análisis y la interpretación de poesía, entre otros (2010: 7).

Entre los resultados preliminares del estudio, du Plooy resaltaba los siguientes: 1) La narratividad como un rasgo preponderante de la poesía lírica contemporánea. 2) Los elementos narrativos - tanto temáticos como estructurales o técnicos- pueden encontrarse tanto en poemas aislados como en ciclos, series y volúmenes de poesía. 3) Existen narrativas subyacentes en las obras poéticas, que pueden ser comunes a conjuntos más amplios, como la poesía de una nación o de un idioma. Así, los poemas pueden ser leídos como parte de narrativas mayores ${ }^{9}$. 4) En la literatura contemporánea, la experimentación genérica es muy habitual; asimismo, las visiones contemporáneas sobre el género literario aprecian que los aspectos genéricos facilitan la comunicación y no deberían ser considerados como prescriptivos (2010: 7-8). Además, du Plooy plantea que: 1) La relación entre lírica y épica debería ser explicada en términos de modos de comunicación en vez de género, por lo que propone una escala gradual más que una distinción neta entre ambos aspectos. 2) El estudio de la narrativa en la poesía lírica debería enfocarse en la variedad y en el alcance de las manifestaciones de la narratividad en los textos líricos más que en las diferencias intergenéricas. 3) Estudiar los aspectos narrativos de la poesía lírica proporciona un enfoque alternativo que conduce a nuevas y apasionantes lecturas de poemas y a nuevas e ingeniosas interpretaciones. 4) Las funciones narrativas son una forma importante de coherencia en poesía. Existe una dinámica específica en poesía, una tensión poética, en la cual la coherencia creada por la narrativa es contrarrestada por

\footnotetext{
${ }^{9}$ Esto entronca con la noción de frame que aplica Hühn y resulta especialmente interesante para el análisis de corpus amplios, ya que las narrativas subyacentes funcionan como elementos de cohesión textual que permiten abordar la unidad de sentido del universo poético de un autor. Como destacaba el narratólogo alemán, sirven como marcos para determinar la plausibilidad de los esquemas; además de como posibles pautas y guías para la interpretación. Estas narrativas pueden afectar a cómo es leída una obra en un determinado periodo; e, incluso, la publicación de un texto puede ser el punto de partida de nuevos relatos que sirvan de marco interpretativo a obras anteriores o posteriores. En el caso del género lírico, ante la escasez de elementos circunstanciales que le caracteriza, la presencia de una narrativa subyacente, bioliteraria, condiciona fuertemente la lectura. Estas narrativas pueden también localizarse en los paratextos de la obra, desde los prólogos hasta los resúmenes o notas biográficas.
} 
el encantamiento poético: "by the lyric and poetic incantative and metaphorically intensifying characteristics" (2010: 9). 5) El desarrollo temporal y secuencial vincula las partes separadas del poema, mientras que la expresión poética intensifica la experiencia emocional del lector. Los aspectos narrativos del poema crean movimiento, mientras que rasgos poéticos como la densidad metafórica, los patrones sintácticos, el ritmo o el metro, constituyen los efectos estéticos. 6) Existe una tensión entre los segmentos narrativos y la segmentación poética, que es una extensión de la existente entre la sintaxis y la tipografía. 7) Las narrativas en los poemas habitualmente son reducidas o incluso elípticas. A menudo un poema contiene únicamente un momento narrativo, pero ese momento efectúa un cambio radical en la narrativa (Hühn, 2009). 8) La relación entre los elementos narrativos y los indicios (elementos poéticos o estéticos) es importante. A diferencia de los textos narrativos, en un poema habrá más elementos poéticos que narrativos. 9) A menudo, la narración en un poema se convierte en una metáfora, y los aspectos narrativos pueden adquirir un sentido metafórico. Una narrativa como la de un mito, una leyenda o un cuento de hadas proporciona al poema un arsenal metafórico. Una historia que es activada intertextualmente en un poema puede proporcionar un conjunto de frames y de scripts ${ }^{10}$. 10) Tanto la teoría de la poesía como la narratología postclásica pueden beneficiarse de este enfoque, que considera más interactivo e integrado; ya que, si los diferentes modos y géneros aparecen mezclados en la práctica poética, los teóricos deberían, según la investigadora, adaptar a ellos sus enfoques teóricos para hacer lecturas más adecuadas de los textos (du Plooy, 2010: 9-10).

\section{NARRATIVA Y POESÍA: SEGMENTACIÓN Y COUNTERMEASUREMENT}

Brian McHale es otro de los principales estudiosos de este enfoque, que ha desarrollado en el marco del proyecto Narrative de la Universidad de Ohio. En 2009 publicaba el artículo "Beginning to Think about Narrative in Poetry". El título (que reconoce "francamente presuntuoso") le sirve

\footnotetext{
${ }^{10}$ Este aspecto ha sido estudiado desde la temática y la tematología; la aparición de un tema en sus múltiples modalidades (argumento, concepto, mito, tópico, asunto, etc.) funciona de elemento de cohesión textual y su emergencia (Brunel) proporciona una pauta interpretativa.
} 
para poner el acento en la escasa atención que se le ha concedido en la narratología más contemporánea a la poesía narrativa. Lo poético sería un ángulo muerto en la teoría narrativa contemporánea, a pesar de que, con anterioridad al siglo XIX, la mayoría de los poemas eran narrativos. Este blind spot sería, en opinión de McHale, el resultado de la especialización institucional y académica. A diferencia de las investigaciones del grupo de Hamburgo y el proyecto de Sudáfrica, que se centran en las convergencias entre narrativa y lírica, McHale se interesa por la relación entre narrativa y poesía, ya que, incluso aquellos poemas de la tradición que son indispensables en la tradición occidental, como los homéricos, "tend to be treated as de facto prose fictions; the poetry drops out of the equation" (2009: 11-12).

Uno de los problemas más frecuentes en el estudio del género es el extendido empleo como sinónimos de los términos lírica y poesía; debido, en buena medida, al predominio casi absoluto de la lírica sobre la épica o la didáctica en la poesía del siglo pasado. McHale propone distinguir entre poesía y lírica, así como entre lirismo y poeticidad. Para ello, se basa en la definición de poesía del "Manifiesto" de Rachel Blau DuPlessis, que considera la segmentación como la característica subyacente del texto poético:

Poetry is the kind of writing that is articulated in sequenced, gapped lines and whose meanings are created by occurring in bounded units precisely chosen, units operating in relation to chosen pause or silence. Thus a fusion of reading, observing, and listening techniques are required to decode it [...]. I propose that segmentativity - the ability to articulate and make meaning by selecting, deploying and combining segments - is the underlying characteristic of poetry as a genre (DuPlessis, 1996: 51).

La segmentación sería así el rasgo dominante del texto poético, que lo diferenciaría del narrativo y el dramático, en los que estaría supeditada a la narratividad y a la performatividad. En la poesía serían centrales los espacios en blanco, que contribuyen a la construcción del sentido. Para McHale, la prosa constituiría el grado cero de segmentación. La poesía en prosa hace que sea significativa la desviación con respecto al verso. Incluso la poesía oral (en la que sería más complejo advertir esta dominante) utiliza, 
según McHale, periodicidades y lapsos: las pausas, el silencio al principio y al final serían el equivalente oral del hueco visual, del blanco (2009: 15).

McHale acude también a una investigación de John Shoptaw "The Music of Construction: Measure and Polyphony in Ashbery and Bernstein" (1995), en la que estudia los diferentes niveles de medida posibles en un poema: en primer lugar, la palabra (una palabra por verso); por debajo del nivel de la palabra, se encontrarían las letras y los signos de puntuación, como sucede en algunos poemas vanguardistas (por ejemplo, el final de Altazor); a continuación, la frase; la línea, la oración y la sección. McHale les añade los niveles de la estrofa y el del pie métrico.

Además de la medida, la poesía se caracteriza, según Shoptaw, también por la contramétrica (countermeasurement): los fenómenos de subversión y ruptura de la medida en diferentes niveles del verso y de la estrofa. El encabalgamiento, por ejemplo, sería uno de los procedimientos que produciría tensión entre las medidas propias de la frase y la del verso.

Esta noción de segmentación es aplicada por McHale a la relación entre narrativa y poesía. Entre las múltiples formas que adopta la segmentación en poesía, distingue aquellas se ubican en el nivel de la historia, como las subtramas o los episodios, y aquellas que pertenecen al discurso, como son las voces yuxtapuestas, los puntos de vista, los distintos tiempos y espacios, etc. Los ejemplos que analiza en su artículo son cuatro diferentes traducciones de un pasaje de la Ilíada, realizadas por George Chapman, Alexander Pope, Richard Lattimore y Maverick Christopher Logue. A través de ellas, explica cómo en cada texto la segmentación narrativa interactúa de una manera diferente con la segmentación poética.

En las conclusiones de su artículo, McHale sugiere lanzar un programa de investigación sobre la narrativa en poesía a partir de estas dos nociones de segmentivity y countermeasurement, con los objetivos de comprender cómo los diferentes metros, esquemas rítmicos y estróficos interactúan con la segmentación propia de la narrativa; y cómo las narraciones han sido manejadas de manera diferente según los géneros y subgéneros; así como en los distintos periodos históricos, tradiciones culturales e idiomas. También, plantea la necesidad de averiguar si han existido estilos distintos de segmentación en función de los periodos y las escuelas (2009: 23). Un trabajo preliminar, señala, sería el estudio de cómo una misma narrativa poética es traducida de un idioma $u$ otro, o de un tipo de verso a otro: 
Filling in these and other lacunae in contemporary narrative theory seems to me a worthy project and a desirable end in itself, if only because it would bring more fully within the scope of narrative theory a huge volume of the world's most valued narratives, namely those written in poetry (McHale, 2009: 23).

La propuesta de McHale ha estado enriquecida por la discusión al respecto con su colega de la Universidad de Ohio, Bruce Heiden, especialista en poesía clásica, en el cruce de réplicas que intercambiaron. Heiden reprocha a McHale la identificación de poesía y verso, y cuestiona su noción de versificación, ligada a su juicio a la discontinuidad. Para Heiden, el verso se caracteriza ante todo por la combinación y continuidad. Critica también Heiden la noción de Shoptaw y McHale de contermeasurement, a la que tacha de anómala en la tradición clásica, pues lo más habitual en esta, señala, es la coincidencia y el refuerzo entre sintaxis y versificación. Asimismo, Heiden objeta también la noción misma de narrativa, que considera una ilusión producida por la narratología estructuralista, ya que no puede existir una narrativa desligada del material verbal concreto del poema y de la situación comunicativa, anclada históricamente del mismo:

An approach to poetry through the lens of narratology confronts an array of misconceptions that block and distort one's view of poetry and of narrative as well. The root of these misconceptions is the fundamental premise that narrative is a linguistic object wholly open to empirical analysis and scientific generalization (Heiden, 2014: 270).

En su lugar, defiende un estudio histórico de las diferentes prácticas $\mathrm{y}$ formas de narración (storytelling) en verso, como dos "synergic dimensions of poetry" (Heiden, 2014: 276).

En su respuesta a Heiden ("Thinking Some More about Narrative in Poetry: A Brief Reply to Bruce Heiden"), McHale vuelve sobre aquellos puntos de discrepancia entre ambos, lo que le permite desarrollar y matizar las ideas centrales de su exposición. Defiende que ambas aproximaciones al verso - ya sea entender la segmentación como interrupción o como continuidad - son dos aspectos complementarios de un mismo fenómeno 
(2014: 285), pues la interrupción implica la existencia de un patrón cuya finalización o repetición es anticipada por el lector. La disparidad no es más que aparente. McHale resalta, por otro lado, que su objeto principal de interés no es la relación del segmento (verso) con la unidad sintáctica, sino con las unidades narrativas: "events, sequences, shifts o voice and focalization, shifts among narrative levels, etc." (2014: 285), es decir, no las distintas formas de segmentación poética, sino propiamente la relación entre la segmentación poética y la narrativa; lo que entronca con el programa de Hamburgo.

En cuanto al segundo argumento de Heiden, respalda el empleo de la noción de narrativa en poesía:

If the category of "narrative" is inappropriate in characterizing "poetic storytelling", how does one know one is in the presence of a story at all, instead of, say, a lyric effusion, a description, or a harangue [...]. Surely there must be something that distinguishes poetic storytelling from other, non-story forms of theme-driven edifying discourse? And if that something isn't what narrative theory calls "narrative", then what is it? (McHale, 2014: 287).

La discusión entre McHale y Heiden ha contribuido a poner el foco y a internacionalizar más, si cabe, este enfoque de estudio. Podemos encontrar ecos, ya desde el propio título, en la investigación de Stefan Kjerkegaard, profesor en la Universidad de Aarhus, en Dinamarca: "In the Waiting Room: Narrative in the Autobiographical Lyric Poem, Or Beginning to Think about Lyric Poetry with Narratology" (2014), centrada en el estudio de los poemas autobiográficos, que plantean un doble interés por la cuestión de la ficción y las narrativas personales.

Esta internacionalización del enfoque narratológico se refleja también en estudios como el de Ludmila Comuzzi (2016), que aborda la problemática de la narratividad de la poesía lírica desde la ciencia cognitiva o Sonja Klimek (2013), quien estudia la figuratividad (en tanto que elementos micro-narrativos de estilo) en la poesía lírica; así como en su aplicación a otras literaturas, además de las ya mencionadas, como por ejemplo la coreana (Yong Ho Choi, 2008).

Además, esta corriente ofrece también espacios por explorar en lo relativo a sus perspectivas intermediales. Brian McAllister, en su 
artículo "Narrative in Concrete / Concrete in Narrative: Visual Poetry and Narrative Theory" (2014), ha emprendido el estudio de la narratividad y sus grados en la poesía concreto-visual, una línea de investigación que parte de las teorizaciones de Marie-Laure Ryan $(2005,2007)$ acerca de la diferencia entre narrativa y narratividad y de Werner Wolf (2003), que abren el camino a la aplicación de las categorías narratológicas en otros sistemas semióticos ${ }^{11}$.

\section{UNMODELOALTERNATIVO: LÍRICAYPERFORMANCE}

Si bien el modelo narratológico de investigación constituye una de las vías más desarrolladas en el panorama internacional de entre aquellas que buscan renovar las coordenadas teóricas de estudio de la poesía lírica, no es, sin embargo, objeto de un consenso unánime en el panorama crítico. A continuación, se presentará otro enfoque teórico que, desde un entendimiento radicalmente distinto de hacia dónde deben dirigirse los estudios de lírica, se establece como otro punto de partida para una teoría general del género.

Se trata de la propuesta de Jonathan Culler, que desarrolla ampliamente en su libro Theory of the Lyric (2015), y que había avanzado en artículos anteriores, como "Why Lyric?" (2014). El aspecto central de esta postura es su rechazo a un enfoque mimético-ficcional de la lírica, que conduce a su asimilación a la narrativa. Considera Culler que, durante el siglo XX, especialmente a partir de los postulados del New Criticism, se ha impuesto en el mundo académico un determinado modelo de entendimiento del poema que lo considera el discurso ficcional de una persona, un tipo de monólogo dramático que exige una interpretación. Esta manera de concebir la lírica produce un tipo de lectura centrada en la búsqueda en el texto de dicho hablante poético (a semejanza del narrador) $\mathrm{y}$ de la reconstrucción de la situación y los motivos de su discurso ${ }^{12}$.

\footnotetext{
${ }^{11}$ Mientras que por narrativa entiende el objeto semiótico, la narratividad consistiría en "being able to inspire a narrative response" (Ryan, 2005: 347).

${ }^{12}$ En España también han existido teóricos que se han posicionado contra esta asimilación. Luján rechaza el traslado a la lírica de las teorías de la ficción a las que condujeron las escuelas del formalismo ruso, el estructuralismo y el New Criticism: "El resultado fue la obligación de entender el discurso lírico como la imitación de un discurso, o cita de un discurso que emite un hablante imaginario, al modo del narrador de una novela" (2005: 23).
} 
En su opinión, este enfoque imperante en la crítica ha tenido como efecto que se hayan descuidado aquellos rasgos más propiamente poéticolíricos, como son sus aspectos rituales (rima, metro, etc.), así como los intertextuales:

There are numerous reasons to resist the model of lyric as dramatic monologue. It pushes lyric in the direction of the novel by adopting a mimetic model and focusing on the speaker as character, but is deadly to try to compete with narrative on terrain where narrative has obvious advantages. This model ignores or reduces, with its normalizing novelizing, the characteristic extravagance of lyric on the one hand and its intertextual echoes on the other, and it neglects all those elements of lyric -including rhyme, meter, refrainnon imitated from ordinary speech acts. It implicitly denies three dimensions of lyric: the effects of presentness of lyric utterance, the materiality of lyric language that makes itself as something other than signs of a character and plot, and the rich texture of intertextual relations that relates it to other poems rather than to worldly events (Culler, 2015: 118-119).

Este rechazo a la asimilación del género no supone, sin embargo, un alineamiento pleno con las teorías que históricamente han defendido en la crítica posturas anti-miméticas, como la de Käte Hamburger, ni una vuelta a una concepción romántica (expresiva) de la lírica, sino que pretende una superación de ambas formas de concebir el poema:

I attempt to work out a general framework, a theory of the lyric, in which attention to these features of lyric is encouraged and nor restricted by one of the narrow models of lyric that have in recent years organized most approaches to lyric poetry (Culler, 2015: VIII).

Propone en su lugar un enfoque alternativo, performático, del cual destacaremos seis elementos o nociones fundamentales que introduce: 1) apóstrofe, 2) ritualidad, 3) memoria, 4) presencia, 5) hipérbole, 6) erótica frente a hermenéutica.

Culler se opone también a la postura de los New Lyric Studies de la no existencia del género y defiende una continuidad del mismo a través 
de dichos aspectos comunes, que analiza en un corpus de nueve poemas de la tradición occidental, desde Safo a John Ashbery.

Desde sus tempranas investigaciones sobre el apóstrofe (1977) como una figura central en la tradición poética occidental, Culler ha desarrollado una concepción de la lírica como un discurso dirigido, que contrasta con la postura bajtiniana que hace de ella el género monológico por excelencia. En su Theory of the Lyric, Culler investiga las modalidades de la apelación lírica (lyric address), especialmente la denominada "triangulated address": un tipo de apelación que se dirige indirectamente a los lectores mediante el procedimiento de dirigirse (o de pretender dirigirse) a una tercera instancia, animada o inanimada (un dios, un amante, fuerzas naturales, etc.). Es, considera Culler, frecuente en la tradición e implica no solamente un mecanismo constructivo sino una manera de concebir el universo característica de la lírica occidental: "The tension between the lyrical positing of an addressable and potentially responsive universe and skepticism about the efficacy of lyric discourse is a determining feature of a wide range of Western lyrics" (2015: 8).

Una de sus manifestaciones más espectaculares, dirá Culler, es la invocación de los ausentes o las apelaciones a lo no humano (2015: 8). La apelación lírica no siempre ha de aparecer explícitamente en el poema, como hemos mencionado, ya que reviste también otras formas indirectas, y subyace en poemas en los que el yo lírico aparentemente no se dirige a nadie.

En segundo lugar, la noción de ritualidad que propone como característica del género ${ }^{13}$. Culler denomina rituales a aquellos aspectos formales del poema relacionados con la performance: el ritmo, la rima, el metro, la repetición, etc. Para el autor, la lírica implica una tensión entre los elementos rituales y ficcionales del poema: "between formal elements that provide meaning and structure and serve as instructions for performance and those that work to represent character or event" (2015: 7). La dimensión formal es la que convierte a los poemas en textos compuestos para su representación (reperfomance) (2015: 37). Esta idea de una tensión en el poema concuerda con la oposición de du Plooy entre aspectos narrativos y estéticos, aunque, mientras que los aspectos narrativos proporcionaban

${ }^{13}$ Culler toma este concepto de la noción de Roland Greene (1991) de una dimensión ritualista de la lírica. 
cohesión frente a la emoción que aportaban los elementos poéticos, en Culler los elementos ficcionales sirven a una función ante todo mimética, relacionada con los personajes y las tramas.

La ritualidad está ligada al tercer rasgo: la lírica como un discurso memorable, destinado a ser recibido, reactivado, repetido en actos de lectura. Su conservación en las memorias individuales y en la memoria colectiva constituiría la garantía de su eficacia en tanto que actuación (performance). Culler toma la idea derridiana del poema que pide ser conservado en la memoria, que dice "apprends moi par coeur, recopie, veille et garde-moi” (Derrida, 1995: 288). En última instancia, ese carácter memorable culmina en su perduración como lugar común o cliché (hay numerosos ejemplos de ello en la poesía española, piénsese en autores como Bécquer o Machado). El poeta escribe "memorable words" (Culler, 2015: 120), a lo que contribuyen los aspectos ritualistas del sonido (melos) y visuales (opsis), a través de los patrones de reiteración del verso, la rima, la distribución espacial, etc.

En cuarto lugar, la lírica como presentación. Los elementos miméticos implican una tensión en el poema debido a que lo propiamente lírico no es la representación, según la teoría de Culler, sino que lo esencial en la lírica ocurre en el poema, en el presente de la enunciación lírica: “A distinctive feature of lyric seems to be this attempt to create the impression of something happening now, in the present time of discourse" (2015: 17).

Este efecto de presencia es lo que hace que el poema esté más próximo a constituir un acontecimiento en sí mismo que una representación de un acontecimiento: "Disrupting narrative, invocation, or address makes the poem an event in the lyric present rather than the representation of a past event" (2015: 8).

Para justificar este punto, Culler vuelve sobre la cuestión central de la polémica: la relación entre el yo lírico y el autor biográfico, y la concepción mimética y/o ficcional de su discurso. Culler no se posiciona de manera plena ni en la tradición teórica romántica que hace de la lírica el género de la subjetividad; y que en el siglo XX han defendido teóricos como K. Hamburger. No adopta íntegramente los postulados de Hamburger, para quien la lírica es un discurso que habla sobre este mundo y no la proyección de un hablante ficticio y un universo ficcional. Sin embargo, sí que hace una relectura de la aportación de esta teórica, que considera malinterpretada. Según Culler, Hamburger no propone una identificación del hablante lírico 
con el autor biográfico, sino que afirma que esta relación es indeterminada. También, señala, en Hegel estaría implícito que la subjetividad lírica es un principio formal de unidad más que la conciencia de un individuo (2015: $350)$.

Para Culler, lo propio de la lírica es la presentación, no la representación (mímesis). Frente a aquellos que la entienden como discurso imitativo, la lírica entronca con el discurso epidíctico de la retórica: "Lyric as epideixis - public discourse about meaning and value- made distinctive by its ritualistic elements. With this conception as starting point, one can then specify that many lyrics incorporate fictional elements $[\ldots]$ " (2015: 350).

Otra de las estructuras características de la lírica es lo que Culler denomina su carácter hiperbólico, es decir, la presunción implícita en el poema de su significación, de su importancia, incluso en aquellos poemas triviales o que no tratan aparentemente sobre nada. El autor propone una escala entre un polo extático —en el que lo hiperbólico; la dimensión trascendente del poema sería manifiesta - y un polo lacónico - en el que existe una aparente trivialidad y lo hiperbólico es una convención subyacente, que funciona por medio del contraste- - "This presumption of significance is what permits one to speak, however counterintuitively, of the hyperbolic character of laconic poems: there is an implicit claim that whatever is lyrically noted is of overweening importance" (2015: 260).

Por último, Culler se opone a una concepción de la lírica como un discurso destinado a producir una interpretación. Frente a la asunción de la crítica moderna de que la finalidad de la lectura de un poema es generar una interpretación, el autor recuerda que en siglos anteriores existían otras posibles formas de recepción, como el análisis, la imitación, la traducción, al memorización, la evaluación o la identificación de alusiones y figuras retóricas (2015: 5). Aboga, como Susan Sontag, por volver a una erótica de la literatura, a una poética que no esté subordinada a la hermenéutica (2015: 6). Sugiere desplazar el eje de estudio de la interpretación al encantamiento, a una erótica del texto en la que ocupen su lugar central las características específicas de la lírica.

Culler no se propone ofrecer una teoría acabada sino, afirma, desplazar el foco de modelos inadecuados y reemplazarlos con uno que permita resaltar aquellos aspectos esenciales de la lírica. Busca abrir caminos y generar nuevos recursos para futuros acercamientos desde lo que 
sería una ampliación del marco teórico, más que una completa sustitución. Defiende que el marco general de la lírica debe reconocer y abarcar la función que desempeñan los elementos ficcionales en el poema, pero manteniendo la primacía de aquellos que no son asimilables a la narrativa (2015: 121). La convención poemática de un hablante ficticio sería en este enfoque una posibilidad entre otras, pero no un modelo general válido para la lírica.

La categoría que permite englobar mejor la propuesta de Culler es la de la lírica como performance. La concepción que le interesa de lo performativo choca con la polisemia actual del término en la crítica literaria. Por ello, distingue entre cuatro sentidos: una performatividad general, relacionada con el sentido austiniano de hacer cosas con palabras, que tiene que ver con la capacidad del discurso literario en general y al ficcional en particular de hacer que algo sea. En segundo lugar, una performatividad básica en la lírica, que no crea un mundo ficcional sino que se autoestablece como lírica. En tercer lugar, performativo hace referencia a la eficacia del poema en producir aquello que describe (Culler propone restringir el término a esta tercera acepción); $y$, por último, la noción de performance como la acción o el acontecimiento lírico, cuya éxito radica en la propia reiteración de la actuación, en hacerse memorable, repetible, representable: "the lyric performance succeeds as it acts iterably through repeated readings, makes itself memorable" (2015: 131). Esta última es la que le interesa como marco general para el género. Culler relaciona este concepto, siguiendo a Barbara Cassin, con el discurso epidíctico. Para ella, performance sería la mejor traducción de este tipo de este género retórico: el discurso concebido como un acto dirigido a persuadir, mover o innovar (Culler, 2015: 131).

\section{CONCLUSIONES}

En este artículo se han examinado dos caminos abiertos actualmente en la crítica internacional para repensar el marco teórico de estudio del texto lírico. Aunque las primeras investigaciones en ambas líneas datan de las últimas décadas del siglo XX, es en el presente siglo cuando se han desarrollado y establecido. Su difusión en el mundo académico contrasta con el escaso eco que han tenido en la teoría literaria en lengua española, ya que apenas existen traducciones, estudios sobre sus aportaciones o 
grupos de investigación que aborden dichas problemáticas, especialmente en el ámbito de la aplicación de la narratología a los textos líricos.

Estos enfoques coinciden, en primer lugar, en su aspecto más programático que sistemático, ya que no se consideran teorías definitivas $\mathrm{y}$ acabadas del género, sino que se asumen como coordenadas nuevas para producir herramientas teórico-críticas en un ámbito, como es el de la lírica, necesitado de nuevos acercamientos. Ambos caminos comparten, en segundo lugar, el reconocimiento de un objeto de estudio (la poesía lírica) frente a las corrientes actuales que niegan la existencia del género con anterioridad al periodo romántico y reducen la investigación a la genealogía de su conformación moderna. En tercer lugar, puede constatarse una pretensión de ampliación del marco teórico, que permita abarcar y superar la dicotomía central en el texto lírico entre sus aspectos miméticos y no miméticos. Si bien hay un posicionamiento distinto en ambos enfoques, se busca un marco general que no niegue otras dimensiones del poema (las más estrictamente poéticas en la corriente narratológica, o la ficcional en el caso de Culler).

A pesar de estas similitudes, se trata de dos vías cuyo foco de interés en el estudio del género es claramente contrapuesto. Mientras que la corriente narratológica propone utilizar las herramientas desarrolladas para el género narrativo a la lírica, partiendo de una concepción imitativa de la literatura en su conjunto, la postura de Culler previene justamente contra este ejercicio de asimilación, al que parece favorecer la hegemonía de la narrativa en la literatura moderna y contemporánea. En la raíz de su diferencia está la vieja cuestión irresuelta de la Poética sobre si la lírica es un género mimético. Su oposición parecería resucitar la polarización recurrente en el siglo XX sobre la lírica como ficción o como expresión, que refiere Fernando Cabo en la introducción a su volumen de Teorías de la lírica ${ }^{14}$. Sin embargo, ambas corrientes se posicionan de tal manera que, sin omitir la problemática, tienden a superarla ampliando el marco. Así, Culler admitirá la existencia de imitación en el poema, pero advertirá de la no prevalencia de estos aspectos ni su validez como base de entendimiento del género. En los estudios de narratología transgenérica también se

\footnotetext{
${ }^{14}$ Las partidarias de la existencia de ficción en la lírica serían "aproximaciones que enfatizan la dimensión representativa, mimética, constructiva o sencillamente conflictiva del propio fundamento enunciativo que tiene el poema lírico" (Cabo, 1999: 13).
} 
explicita la preocupación por no descuidar los rasgos más específicamente poéticos, sobre todo los formales, e incluso hacer de la tensión entre segmentación poética y narrativa una de las claves del nuevo ámbito de investigación (McHale). Se aprecia una pretensión de integración más que de reducción, para lo cual son herramientas útiles el establecimiento de escalas graduales entre géneros y de polaridades ${ }^{15}$. Mediante su desarrollo de aspectos contrapuestos del género, ambas corrientes contribuyen a un más amplio entendimiento de la complejidad textual del poema y de las múltiples posibilidades de combinación en él de las distintas funciones lingüísticas, una perspectiva no reduccionista ya esbozada en las primeras teorizaciones formalistas del poema como complejo rítmico-semántico en el que coexisten leyes distintas.

La investigación narratológica sobre textos poéticos y líricos ha avanzado notablemente en las dos últimas décadas, pero se encuentra todavía en un estado incipiente en cuanto a su aplicación crítica en las diferentes literaturas y en los subgéneros históricos. En las literaturas en lengua española, como se ha mencionado antes, apenas se han realizado estudios desde este enfoque, pese a que, a su favor, se hallaría la presencia de una rica tradición de poesía narrativa, así como nuevas formas de hibridación en la modernidad, como los Poemas encadenados de Pedro Casariego Córdoba. En la lírica contemporánea, marcada por el llamado giro narrativo, también permanecen muchos espacios por explorar, así como la cuestión de las narrativas subyacentes, tanto nacionales como personales, especialmente en la poesía de tipo autobiográfico o confesional, ampliamente practicada tanto por la tradición de la poesía de la experiencia como por poetas emergentes, sin olvidar las formas de autoficción lírica. La corriente narratológica se muestra especialmente relevante, además, para abordar críticamente experimentos de transgresión genérica, a partir de la noción de narratividad en los textos líricos.

Las aportaciones de los estudios del grupo de Hamburgo y de Sudáfrica cartografían un terreno cuya exploración (en las literaturas nacionales, así como en los subgéneros históricos) está pendiente de realizar.

La adopción de las coordenadas teóricas de la narratología

${ }^{15}$ En España, Francisco Javier Ávila proponía en 2006 esta forma gradual de entender las diferencias entre géneros, mediante un sistema de polos y ámbitos. 
transgenérica, pese a estas nuevas y florecientes vías abiertas de investigación, no debería generalizarse irreflexivamente, ya que puede suponer una asimilación que descuide los aspectos puramente poéticos y líricos, algo contra lo que advierte Jonathan Culler.

A su vez, las nociones introducidas por el teórico de Cornell proporcionan también líneas no acostumbradas de investigación del género, rompiendo con ciertos lugares ya comunes de entender la lírica, por ejemplo su condición de discurso monológico o la hegemonía de la interpretación, y resultan de una gran relevancia para abordar las manifestaciones de poesía contemporánea más cercanas a la oralidad, desde la poesía que se recita en las salas de jam session actuales hasta las tendencias de poesía sonora y videopoesía. Su vuelta a una vinculación con el género epidíctico, la ritualidad y la performance establece unas coordenadas de partida para un renovado marco teórico y unas herramientas críticas ad hoc para la poesía lírica.

\section{REFERENCIAS BIBLIOGRÁFICAS}

ALBER, J. \& FLUDERNIK, M. (2013). "Mediacy and Narrative Mediation". En The living handbook of narratology. Hamburg University Press, https://wikis.sub.uni-hamburg.de/lhn/index.php/ Mediacy and_Narrative_Mediation [11/12/2018].

ÁVILA GONZĀLEZ̄, F. J. (2006). "Polos y ámbitos para una teoría de los géneros. El polo lírico frente al polo narrativo". Analecta Malacitana 29.1, 71-111.

BURT, S. (2016). "What Is This Thing Called Lyric?”. Modern Philology 113.3, 422-440.

CABO ASEGUINOLAZA, F. (1999). Teorías sobre la lírica. Madrid: Arco / Libros.

CALLES MORENO J. M. (2002). La modalización en el discurso poético. Tesis doctoral: Universitat de València. Versión digitalizada por la Biblioteca Virtual Miguel de Cervantes: http://www. cervantesvirtual.com/obra/la-modalizacion-en-el-discursopoetico--0/[11/12/2018]).

CARRIZO RUEDA, S. M. (2014). "Poesía lírica y teoría crítica del siglo XX. Entrevista a Miguel Ángel Garrido Gallardo”. Letras 69/70, 
133-150.

CHAIX-BRYAN, T. (2008). "Lyrisme et narratologie". Acta Fabula 9(3) (también en http://www.fabula.org/acta/document3976.php [11/12/2018]).

COMUZZI, L. (2016). "Lyric Poetry as a Narrative Speech Genre: On the dialogue between genre theory and cognitive science". Amsterdam International Electronic Journal for Cultural Narratology 7/8, 51-73 (también en http://cf.hum.uva.nl/ narratology/issue/7 /a12_ Ludmila_Comuzzi.html [11/12/2018]).

CULLER, J. (1977). “Apostrophe”. Diacritics 7.4, 59-69. (2014). "Why Lyric?”. PMLA 123.1, 201-206.

(2015). Theory of the Lyric. London: Harvard University Press. (2016). "Poetics, Fictionality, and the Lyric". Dibur 2, 19-25 (también en https://arcade.stanford.edu/dibur/poetics-fictionalityand-lyric-0 [11/12/2018]).

DERRIDA, J. (1995). Points... Interviews, 1974-1994. Standford, California: Standford University Press.

DUBROW, H. (2006). "The interplay of narrative and lyric: competition, cooperation, and the case of anticipatory amalgam". Narrative 14.3, 254-271.

DUPLESSIS, R. B. (1996). "Manifests". Diacritics, tomo 26.3-4, 31-53.

DU PLOOY, H. J. G. (2010). "Narratology and the study of lyric poetry". Literator 31. 3, 1-15.

GARCÍA BERRIO, A. (1978). "Lingüística del texto y texto lírico. La tradición textual como contexto". Revista española de lingüística 8.1, 19-76.

(1989). Teoría de la literatura: la construcción del significado. Madrid: Ediciones Cátedra.

GREENE, R. (1991). Post-Petrarchism. Nueva Jersey: Princeton University Press.

HAMBURGER, K. (1995). La lógica de la literatura. Madrid: Visor Libros.

HEIDEN, B. (2014). "Narrative in Poetry: A Problem of Narrative Theory". Narrative 22.2, 269-283.

HO CHOI, Y. (2004). “Transgeneric Narratology: Applications to Lyric Poetry". En The Dynamics of Narrative Form, J. Pier (ed.), 139158. Berlin: de Gruyter. 
(2005). "Plotting the lyric: forms of narration in poetry". En Theory Into Poetry: New Approaches to the Lyric, E. Müller-Zettelmann y M. Rubik (eds.), 147-172. Amsterdam-Nueva York: Rodopi. (2008). "Narrative approaches to lyric poetry: On Kim Sowöl's The Azaleas". The Public Journal of Semiotics II.1, 2-10.

HÜHN, P. \& SCHÖNERT, J. (2005). "Introduction: The Theory and Methodology of the Narratological Analysis of Lyric Poetry". En The Narratological Analysis of Lyric Poetry: Studies in English Poetry from the $16^{\text {th }}$ to the $20^{\text {th }}$ Century, P. Hühn y J. Kiefer (eds.), 1-14. Berlín: Walter de Gruyter.

HÜHN, P. \& SOMMER, R. (2012). "Narration in Poetry and Drama", The living handbook of narratology. Hamburg: University Press, http:// www.lhn.uni-hamburg.de/article/narration-poetry-and-drama [17/11/2018].

JACKSON, V. (2005). Dickinson's Misery: a Theory of Lyric Reading. Nueva York: Princeton University Press. (2008). "The New Lyric Studies". PMLA 123, 181-234.

JACKSON, V. \& PRINS, Y. (2014). The Lyric Theory Reader: A Critical Anthology. Baltimore: Johns Hopkins University Press.

KJERKEGAARD, S. (2014). "In the Waiting Room: Narrative in the Autobiographical Lyric Poem, Or Beginning to Think about Lyric Poetry with Narratology". Narrative 22.2, 185-202.

KLIMEK, S. (2013). "Functions of Figurativity for the Narrative in Lyric Poetry - with a Study of English and German Poetic Epitaphs from the 17th Century". Language and Literature 22.3, 219-231.

KRAAN, M. (1991). "Towards a Model of Lyric Communication: Some Historical and Theoretical Remarks". Russian Literature 30, 199230.

LEVY, D. (1988). Chinese Narrative Poetry. The Late Han through T'ang Dynasties. Durham: Duke University Press.

LUJÁN ATIENZA, A. L. (2000). "La lírica y los límites de la ficción”. Revista de Literatura 62, 5-19.

(2005). Pragmática del discurso lírico. Madrid: Arco / Libros.

MCHALE, B. (2009). "Beginning to Think about Narrative in Poetry". Narrative 17.1, 11-27.

(2014). "Thinking Some More about Narrative in Poetry: A Brief reply to Bruce Heiden". Narrative 22.2, 284-287. 
MCALLISTER, B. (2014). "Narrative in Concrete/ Concrete in Narrative: Visual Poetry and Narrative Theory". Narrative 22.2, 234-251.

POZUELO YVANCOS, J. M. (1994). La teoría del lenguaje literario. Madrid: Cátedra.

(1998). “Enunciación lírica?”. En Teoría del poema: la enunciación lírica, F. Cabo Aseguinolaza y G. Gullón (eds.). Diálogos Hispánicos 21, 41-76. Amsterdam-Atlanta: Rodopi. PRINS, Y. (1999). Victorian Sappho. Nueva York: Princeton University Press.

RYAN, M. L. (2005). "On the Theoretical Foundations of Transmedial Narratology". En Narratology beyond Literary Criticism: Mediality, Disciplinarity, J. Ch. Meister (ed.), 1-23. Berlín: de Gruyter. (2007). "Toward a Definition of Narrative". En The Cambridge Companion to Narrative, D. Herman (ed.), 22-35. Cambridge: Cambridge Univversity Press.

SEEMANN, K. D. (1984). "Die Kommunikationsstruktur im lyrischen Gedicht”. En Text, Symbol, Weltmodell: Johannes Holthusen zum 60. Geburtstag, W. Schmid y R. Döring-Smirnov (eds.), 533-554. München: Sager.

STILlingER, J. (1988). "The Plots of Romantic Poetry". College Literature 15 (3), 208-223.

VAN DER ENG, J. (1989). "Narrative Aspects in Puškin's Lyrical Poetry". Russian Literature 26, 441-450.

WESTSTEIJN, W. G. (1989). "Plot Structure in Lyric Poetry: An Analysis of Three Exile Poems by Aleksandr Puškin". Russian Literature 26, 509-522.

WOLF, W. (2003). "Narrative and Narrativity: A Narratological Reconceptualization and Its Applicability to the Visual Arts". Word and image 19.3, 180-197.

(2005). "The Lyric: Problems of Definition and a Proposal for Reconceptualization". En Theory Into Poetry: New Approaches to the Lyric, E. Müller-Zettelmann y M. Rubik (eds.), 21-56. Amsterdam-Nueva York: Rodopi.

Recibido el 8 de enero de 2019.

Aceptado el 14 de marzo de 2019. 